of their production and the early appearance of growth factor receptors in embryogenesis ${ }^{9}$ support a popular prejudice that the serum growth factors, scrutinized so thoroughly within the context of tumour biology, may play pivotal roles in developmental growth and morphogenesis.

The new twist provided by Seifert et al. is the suggestion that 'embryo-derived growth factors' function normally in the autocrine mode. On first consideration the concept is aesthetically distasteful. Why should a developing tissue secrete a growth factor to stimulate its own proliferation? Surely the process could be controlled more stringently from within the cell at some point subsequent to the events governed by growth factors and their receptors. A teleological rationale may be contained within the biological need to amplify weak chemical signals - the raison d'etre of membrane-bound receptors for hormones and growth factors. It is worth noting that normal embryo fibroblast cultures typically require less serum for growth than do established lines of the 3T3 genre, an observation consistent with the view that embryonic cultures respond to the growth factors they produce. Furthermore, another growth factor, interleukin2 , seems normally to function in the autocrine mode ${ }^{10}$.

The interface between growth factors and development remains even less than tenuous. Nonetheless, if recent observations can be extended, the implications are wide indeed. Growth factor receptors and other components of the 'mitogenic cascade' may be vestiges of an automitogenic apparatus used in development. By switching off the gene encoding an automitogen and activating the same gene at some distal site, the developmental machinery may be neatly converted to the paracrine mode. In this state, it could promote cell proliferation 'on demand' during adult life in, for example, wound healing, erythropoiesis and immunogenesis. Switching errors in adult life would manifest themselves as proliferative disorders - keloids, psoriasis, atherosclerosis and, of course, neoplasia.

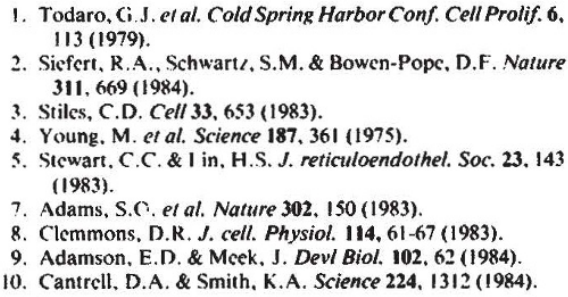

1. Todaro, Gi.J. el al. Cold Spring Harbor Conf. Cell Prolif. 6. 113 (1979).

2. Siefert, R.A. Schwart/, S.M. \& Bowen-Pope, D.F. Nature 311, $669(1984)$

Stiles, C.D. Cell 33, 653 (1983)

4. Young. M. et al. Science 187, 361 (1975)

Stcwart. C.C. \& I in, H.S. J. reticuloendothel. Soc. 23. 143 (1983).

Adams, S.C. et al. Nature 302, 150 (1983).

8. Clemmons, D.R. J. cell. Physiol. 114, 61-67 (1983).

9. Adamson, E.D. \& Mcek, J. Devl Biol, 102, 6? (1984)

10. Cantrcll, D.A. \& Smith, K.A. Science 224, 1312 (1984).

C.D. Stiles is in the Department of Microbiology and Molecular Genetics, Harvard Medical School and the Dana-Farber Cancer Institute, Boston, Massachusetts 02115.

\title{
Biophysics
}

\section{Many guises in Bristol}

\section{from Maxine Clarke}

There probably never was an adequate definition of biophysics. Perhaps there never will be. That, at least, seemed to be one inevitable conclusion of the 8th International Congress of Biophysics held in Bristol, 29 July - 4 August, at which it became clearer than ever just how many biological questions have recently been annexed by biophysicists.

For example, T. Wiesel (Rockefeller University) presented one classic physiological study in the guise of biophysics in reporting how electron microscopy has been used to demonstrate axonal spread of peroxidase-stained neurones throughout several hierarchical levels of organisation in the visual cortex. Physiological experiments on monkey photoreceptors (D. Baylor, Stanford University) show that both rods and cones have identical properties to those deduced by psychophysical studies in man. Many other biological questions, from how ion channels are gated to how microtubules cause cilia to beat, were similarly addressed in biophysical fashion.

To anyone with a primary interest in the structure of cells and organelles, it was very obvious that improvements in electron microscopy and synchrotron radiation measurements had contributed greatly to progress since the 7th congress, three years ago. An optical diffraction pattern taken from an electron microscope image contains information about the phases as well as the amplitudes of the reflections. This inherent advantage of electron microscopy over X-ray diffraction techniques has been limited by problems in the resolving power of the method, but the use of low electron dose to eliminate beam damage to the specimen and the development of cryomethods which do not require staining of the specimen are solving some of the problems. Such improvements enabled E. Kellenberger (University of Basel) to show that the bacterial cell envelope has a periplasmic gel of regular thickness between the inner and outer membranes, with the nucleoids (bacterial DNA) much freer than originally thought. The dependence of nucleoid structure on environmental conditions is now being measured by a freezesubstitution technique. J.L. Carrascosa (Universidad Autónoma de Madrid) produced a $2.2 \mathrm{~nm}$-resolution threedimensional image of the head-to-tail connection (neck region) of the 029 bacteriophage by tilting a two-dimensional hexagonal array of necks and performing a Fourier analysis of differently angled views of the section. The inner region of the neck was identified as the part interacting with the DNA; it has 6-fold symmetry and the proteins constituting this and the 12 -fold outer neck region have been assigned.

Improvements in synchrotron radiation techniques have enabled some of the predictions of the crossbridge theory of muscle contraction to be confirmed for the first time. A two-dimensional detector allows a difference pattern between relaxed and contracting muscle to be produced. $\mathbf{H}$. Huxley (MRC laboratory of Molecular Biology, Cambridge) reported an increase of the actin (thin filament) reflections on contraction; this result is the necessary outcome of the attachment of crossbridges from the thick to the thin filaments but had not been visualized previously. His group has also found that the part of the diffraction pattern that arises from the calcium-binding proteins changes before the part that is related to the crossbridges. This confirms the hypothesis that calcium binding has a structural consequence which must express itself in the muscle filaments before the crossbridges can produce force. In a different approach to elucidating the mechanism of contraction, Y. Goldman (University of Pennsylvania) described an ambitious attempt to link the biochemical rate constants of the muscle's ATPase, derived from solution studies of muscle proteins, with the force-producing properties of the fibres. Caged ATP, an inert molecule which can be photolysed to ATP with UV light after its infusion into a muscle fibre, is used in this approach. Several of the kinetically intermediate reactions in the ATPase in muscle fibres are similar to those in solution, so that the particular step in the biochemical pathway affected by force can now be sought.

Innumerable other topics, ranging from atomic interactions in proteins to the role of biophysics in university teaching courses, were discussed at the meeting. The main virtue of the conference lay in the manner in which a review of a huge number of topics, via sessions centred around either a biological problem or a physical technique, was arranged. This was particularly valuable for highlighting old problems which might now be solved using new methods. Individual sessions did not necessarily provide a balanced view of the field under discussion, but that is always likely to remain the case at the International Congress. The annual meeting of the US Biophysical Society attracts universal attendance and is established as the forum for comprehensive discussions in a large number of subjects. This is more difficult to achieve in a nomadic meeting in which there is a necessarily random sampling of a much wider population. However, for those biophysicists with time and desire to expand their education, looking for a new problem or interested in new methods, the International congresses remain a valuable venue.

Maxine Clarke is an assistant editor of Nature. 